\title{
Review of: "Impact of early corticosteroids on 60-day mortality in critically ill patients with COVID-19: A multicenter cohort study of the OUTCOMEREA network"
}

\author{
Celalettin Ustun, Sunita Nathan
}

Potential competing interests: The author(s) declared that no potential competing interests exist.

This is a retrospective analysis on the prospectively collected data from multi-centers $(n=11)$. The objective of the studies were the effect of early corticosteroids use (CS) in COVID-19 pneumonia patients admitted to the ICU regarding of efficacy (60-day mortality) and safety [ ICU-acquired-bloodstream infections (ICU-BSI), and hospital-acquired pneumonia and ventilator-associated pneumonia (HAP-VAP)]. The authors first found that The authors noted that there was no impact of early CS use (within 48h of ICU admission) on the mortality from COVID19 associated pneumonia when specifically comparing this group with later use of steroids. They also did not find any difference in the associated morbidity from ICU-BSI and HAP-VAP as were similar in the two groups. The difference was found in an adhoc analysis: early CS treatment was associated with a lower mortality rate in patients aged 60 years or more (IPTWHR, 0.53;95\% $\mathrm{Cl}, 0.3-0.93 ; \mathrm{p}=0.03)$. In contrast, CS was associated with an increased risk of death in patients younger than 60 years without inflammation on admission ( ${ }_{\text {PTWW }} \mathrm{HR}=5.01 ; 95 \% \mathrm{Cl}, 1.05,23.88 ; \mathrm{p}=0.04$ ).

The tables provided are comprehensive and along with the forest-plot, representation of the outcomes do provide meaningful information in the early CS and non-early CS groups. However, to me, the most important one would have been the one describing/comparing early CS vs. no early CS use stratified by age (60 and older vs. younger than 60 years).

One limitation that remains is the fact that the number of patients compared in each sub-group is small. Table S4 delineates this clearly. The number of patients in early CS older $\geq 60$ is very limited, $\sim 30$. About half of them had inflammation and the other half no inflammation. Therefore, the main finding of the study that early CS use can reduce mortality was derived from a comparison of two groups of about 15 patients. The readers have to decipher this data from a table, which makes it difficult to see the absolute impact.

Moreover, there are many subjective decisions that were made that can induce biases as noted below: 1-Patient population: 
1. Patients admitted to ICU were included; however, these patient were in various clinical severity regarding respiratory dysfunction and thus required various levels of respiratory support; which included as little as supplemental $\mathrm{O} 2$ to invasive mechanical ventilator support.

2. More of the early CS patients had severe ARDS

3. The impact of associated comorbidities and immunosuppressive status on ICU transfer was not evaluated. These may also affect the potential risk of ICU-BSI and HAP-VAP.

4. Patients who had prior steroids or transferred from other ICUs were excluded (an arbitrary decision).

\section{2-Treatment:}

There was no predefined indication for using CS or specifications on the dose or type of CS. Therefore, patients received CS for many different reasons.

\section{3-Definitions:}

1. Early (within $48 \mathrm{~h}$ of ICU admission) CS use - is not standard because ICU admission criteria was not standard. The definitions prior to data analysis should be more "concrete", potential example to consider CS use 7 days before or after start of symptoms as shown in (Table day-60 mortality) that shows no impact of early CS use.

2. Inflammatory or non-inflammatory (e.g, CRP at the level of 100 was considered high, but not at the level of 99)

Although this study contributes to the literature and our knowledge with prospectively collected data and has sound statistical analyses, the results should be taken carefully because of its retrospective analysis inherited biases mentioned above.

We have also published our experience, in which steroid use was associated with high mortality in allogeneic hematopoietic cell transplantation. ${ }^{1}$ However, when we published, it was unknow that whether this resulted from steroid use or the conditions requiring CS use at the time of Covid-19 occurred. In our second analysis, case series (submitted), we described our experience in which we saw recurrent episodes of inflammatory flares with primarily progressive respiratory symptoms (sometime two or three episodes) which improved their clinical condition with use of CS in real time with these patients.

1-Ankur Varma, Satyajit Kosuri, Celalettin Ustun, Uroosa Ibrahim, Jonathan Moreira, Michael R. Bishop, Sunita Nathan, Jayesh Mehta, Daniel Moncayo, Joseph Heng, Keren Osman, Kehinde U. A. Adekola COVID19 infection in hematopoietic cell transplantation: age, time from transplant and steroids matter. Leukemia. 2020 Aug 14 : 1-4. doi: 10.1038/s41375-020-01019-x [Epub ahead of print] 
